\title{
Propuesta de un nuevo modelo: Práctica Reflexiva Mediada
}

\section{Proposal for a new model: Mediated Reflective Practice}

\author{
Ingrid Eugenia Cerecero Medina ${ }^{1}$ \\ ${ }^{1}$ Centro de Enseñanza de Lenguas, Universidad Autónoma del Estado de México, México (ingridecm@yahoo.com)
}

Recibido el 4 de diciembre de 2017; revisado el 4 de mayo de 2018; aceptado el 7 de mayo de 2018; publicado el 1 de junio de 2018

\section{RESUMEN:}

Esta investigación se focalizó en encontrar la pertinencia de la combinación de elementos para que docentes en activo y con experiencia lleven a cabo ciclos de práctica reflexiva aplicados a su propia labor como medio de mejora y evaluación de la misma.

Se tomaron como punto de partida tres fundamentos para la propuesta: 1) La relación dialógica entre la teoría y la práctica, 2) algunos modelos y características de la práctica reflexiva mencionadas por distintos autores y 3) la relevancia del apoyo de la mediación.

Se exponen, en un primer momento, la importancia de la teoría y la práctica para el análisis de la labor docente, seguido de algunas de las características principales de la práctica reflexiva; algunas notas relevantes sobre la teoría sociocultural de Vygotsky, la mediación y el modelo propuesto por Engeström para la teoría de la actividad; así como la presentación de ciertos modelos representativos de la práctica reflexiva dando como resultado la propuesta de un nuevo modelo al que hemos llamado Práctica Reflexiva Mediada, resumiendo los elementos que lo constituyen y su conceptualización.

El modelo fue aplicado a docentes de lenguas por medio de un taller y el seguimiento de registros y observaciones de su práctica en un período de dos meses. Tiempo durante el cual los docentes tuvieron la oportunidad de interactuar y llevar a la práctica cada uno de los estadios del modelo, los cuales se dividen en tres: conocimiento, significación y resignificación, siendo acompañados de un mediador y sus pares.

Entre los resultados encontrados se destaca la importancia de la participación de un mediador que apoye dicho proceso y el compromiso de los docentes para llevarlo a cabo, reconociendo que las experiencias y saberes de los docentes pueden compartirse y documentarse originando la posibilidad de encontrar nuevos supuestos a comprobar.

\section{PALABRAS CLAVE: TEORÍA Y PRÁCTICA; PRÁCTICA REFLEXIVA; MEDIACIÓN; SIGNIFICACIÓN; TRANSFORMACIÓN.}

\begin{abstract}
:
This research focused on finding the relevance of the combination of elements as support for active and experienced teachers to carry out reflective practice cycles applied to their own work as a means of improving and evaluating it.

Three foundations for the proposal were taken as a starting point: 1) The dialogical relationship between theory and practice, 2) some models and characteristics of reflexive practice mentioned by different authors; and 3) the relevance and support of mediation.

At first, the importance of theory and practice for the analysis of teaching work is exposed, followed by some of the main characteristics of reflexive practice; some relevant notes on the sociocultural theory of Vygotsky, the mediation and the model proposed by Engeström related to the theory of activity; as well as the presentation of certain representative models of reflective practice, resulting in the proposal of a new model that we have called Mediated Reflective Practice, summarizing the elements that constitute it and its conceptualization.

The model was applied to language teachers through a workshop and the follow-up of records and observations of their practice in a period of two months. Time during which the teachers had the
\end{abstract}


opportunity to interact and carry out each of the stages of the model, which are divided into three: knowledge, meaning and resignification, being accompanied by a mediator and their peers.

Among the results, it is highlighted the importance of the participation of a mediator that supports this process and the commitment of the teachers to carry it out, recognizing that the experiences and knowledge of teachers can be shared and documented, arising the possibility of finding new assumptions to be tested.

\section{KEYWORDS: THEORY AND PRACTICE, REFLECTIVE PRACTICE, MEDIATION, SIGNIFICATION, TRANSFORMATION.}

\section{INTRODUCCIÓN}

El artículo resume la investigación titulada "Teorización de los procesos de resignificación de la práctica educativa del docente de lenguas" presentada como tesis doctoral por la autora (Cerecero Medina, 2016), enfocándose en la trascendencia de la práctica reflexiva e identificando la pertinencia de la mediación para llevarse a cabo.

La práctica reflexiva tiene como propósito fundamental la comprensión y mejora del profesionista que la aplica. En el caso del profesorado, se busca que los docentes logren resignificar su práctica, empoderándolos con nueva información que ellos mismos descubran e investiguen y que les permita estar en una constante prospección de elementos y hallazgos a favor de una mejora personal y profesional continua.

El problema que planteamos surge del reconocimiento de la complejidad que los procesos de enseñanza-aprendizaje implican para cada docente y de la formación y recursos que los profesores van obteniendo a lo largo de su carrera; mismos que los van definiendo, muchas veces sin redefinirse conforme se presentan cambios y sin darse cuenta de las consecuencias que esto provoca en su enseñanza. Esto es, uno de los problemas identificados es que los docentes actúan de acuerdo con lo que han aprendido y experimentando, cayendo en ocasiones en la rutina $y$ en cierta falta de consciencia en cuanto a sus acciones en el aula.

Por otra parte, Zeichner (1982) y Carr (1996) señalan que existen docentes que se dejan llevar por las teorías actuales y las tendencias pedagógicas sin detenerse a reflexionar sobre la pertinencia de las mismas en su trabajo. Es decir, actúan de manera acrítica; o en el caso contrario, como lo menciona Carr (1996), existen profesores que actúan por sentido común, según las necesidades que se les presentan, pero sin contrastar lo que hacen con la teoría existente.

El objetivo de la investigación fue capacitar a un pequeño grupo de docentes participantes para llevar a cabo un ciclo de práctica reflexiva, a través del modelo propuesto y conocer, analizar e interpretar los resultados alcanzados.

El sustento teórico de esta investigación se fundamenta en tres ejes: la relación teoría-práctica, la práctica educativa reflexiva y la mediación.

\subsection{Relación Teoría-Práctica}

La relación teoría-práctica es un factor fundamental en la práctica reflexiva puesto que, si se considera únicamente la teoría o la práctica, su análisis será incompleto. Tanto la teoría como la práctica tienen una relación dialógica en la que ambas se necesitan para generarse, comprobarse o corregirse. Autores como Kemmis (1988) y Carr (1996) concuerdan en que la práctica tiene un valor, un significado y un sentido que se construye a partir del actor y el medio en el que se encuentra. De igual manera, la teoría tiene un valor un significado y un sentido que construye el escritor a través de sus investigaciones en un contexto y tiempo determinado. Además de estos autores, Schwab (1983), Tardif (2004) y Gadamer (1980) retoman en sus explicaciones a la filosofía práctica de Aristóteles reconociendo como acciones humanas a la theoría, la poesis y la poiesis, a cada una de las cuales les corresponde un tipo de razonamiento: episteme (teórico), techne (técnico) y phronesis (práctico) respectivamente. Es decir, en la práctica reflexiva se requiere de la teoría, de la técnica y de la práctica, pero además se necesita de una parte reflexiva y crítica que permita un correcto análisis de la misma.

Aun cuando los docentes utilicen los supuestos teóricos provenientes de distintas investigaciones, estos son interpretados de acuerdo con sus propias creencias, experiencias y contexto; de ahí que coincidiendo con Latorre (2003) se enfatiza que los profesores deben asumir el papel de investigadores de manera que se genere un diálogo ente la teoría y la práctica que les permita identificar, analizar y responder a los problemas educativos que se les presenten.

Es necesario reconocer que la teoría educativa lleva una carga política considerándose, por un lado, como una necesidad para sostener y dar continuidad al progreso de una sociedad y por otro, como una forma de proteger los intereses del Estado y tener el control sobre la población, como lo afirman Dewey 
(1995), Delval (1999), Kemmis (Carr, 1996), Aguilar y Viniegra (2003).

Es por ello, que la conexión entre la teoría y la práctica también se relacionan con el currículo, puesto que este es establecido y no elegido por los docentes. Esto es, el Estado decide lo que los alumnos deben aprender, incluyendo aquellos que se están formando para ser profesores. Lo cual trae como consecuencia una enseñanza masificada que incluye a los futuros profesores quienes son preparados por el Estado para que ellos reproduzcan sus aprendizajes de la misma forma en que los adquirieron y que van de acuerdo con lo que el gobierno espera de ellos.

Por supuesto, también existe una teoría independiente que busca ser liberadora y que es producida por aquellos que están preparados para investigar y reflexionar sobre los temas que tratan. Ese es el tipo de teoría que buscamos se tome en cuenta y contraste en la práctica reflexiva.

Los docentes deben estar conscientes de que la teoría es parte de su aprendizaje y que los significados que le otorgan van de acuerdo con sus propios esquemas conceptuales y la interpretación que cada uno de ellos le da, por lo que la teoría debe ser contrastada con lo que sucede en el aula, sin que por ello tenga que ser forzosamente aceptada o rechazada, sino más bien, debe ser probada, analizada y en su caso, adaptada al contexto, enriqueciéndola con las propias experiencias obtenidas.

\subsection{Práctica Reflexiva}

A partir de la revisión de teorías y de observar su práctica, los docentes pueden iniciar un proceso reflexivo.

La relevancia de la reflexión ha sido mencionada desde tiempos remotos (Atkinson, 2011). Por ejemplo, Lao Tsé ya mencionaba que, para lograr el Tao, uno de los puntos era llevar a cabo acciones reflexivas. Sócrates propuso su método socrático en el que establece la importancia del debate, la discusión y el cuestionamiento. Platón resaltaba que el conocimiento verdadero procede de la razón; Aristóteles afirmaba que descubrimos la realidad a través de la observación; y Descartes por medio de su método cartesiano de la duda sugiere poner en entredicho todo lo que se dice o lee. Husserl (García, 1999), en su fenomenología trascendental menciona que cada sujeto le otorga un sentido a su mundo según las representaciones que crea de él, por lo tanto, para reflexionar se debe tomar una actitud científica, sin hacer ningún juicio (epojé). Ya en el siglo XX, John Dewey (1995) estudia el pensamiento reflexivo y define la reflexión como "el discernimiento de la relación que existe entre lo que tratamos de hacer y lo que ocurre como consecuencia" (p. 128). Más adelante encontramos que Berté (2014) divide la reflexión en concomitante y subsiguiente, las cuales son equivalentes a la conciencia pre-reflexiva y la conciencia reflexiva, mencionadas por Sartre (Gallagher \& Zahavi, 2013).

La práctica reflexiva va más allá de una reflexión aislada, constituye como su nombre lo indica una práctica o proceso continuo de reflexiones intencionales y llevadas a cabo de forma sistemática.

Se atribuye a Schön (1992) el término de práctica reflexiva resaltando la existencia de una reflexión en la acción y otra, la reflexión sobre la acción. La primera es similar a la conciencia pre-reflexiva pues se refiere al pensamiento que se tiene sobre la acción en el momento que está ocurriendo y la segunda se refiere a la reflexión que se hace posterior a la acción. Entre los autores que definen la práctica reflexiva, encontramos a Barnett (1992), quien la explica como un procedimiento de formación para:

[...] estimular a los estudiantes a que desarrollen la capacidad de observarse a sí mismos y de emprender un diálogo crítico con ellos mismos en relación con todo lo que piensan y hagan [...] es un procedimiento reflexivo en el que el alumno/a se interroga sobre sus pensamientos y acciones [...] (p. 198).

La definición de Barnett, puede ser aplicable a los docentes si cambiamos la palabra "estudiantes o alumno(a)" por la de "profesores o docente". Considero esta definición puede completarse tomando en cuenta otros elementos en el diálogo crítico que menciona como son la teoría, las experiencias previas del docente, sus conocimientos, y el contexto en que se encuentra.

Entre los distintos autores que han escrito sobre la reflexión o la práctica reflexiva encontramos a Dewey (1993, 1995), Argyris (1980), Schön (1998), Zeichner (1982), Kemmis y McTaggart (1988), Barnett (1992), Latorre (2003), Mertler (2009), Perrenoud (2007), Whitehead (2008), Korthagen (2001), Domingo y Gómez (2014), Esteve (2011), Pedroza, et. al. (2014) y Anijovich (2009), cada uno de ellos expone algunas características coincidentes o que se pueden complementar y que son o debieran ser incluidas en el proceso, de esta manera la práctica reflexiva debe ser un proceso consciente, intencional, metódico, deliberativo, ético, social e histórico, responsable, retrospectivo y prospectivo; busca el desarrollo profesional y humano, implica un distanciamiento o pausa de la actividad para pensar 
en la misma y una suspensión del juicio para actuar de forma objetiva, va encaminado a la intervención, requiere de tiempo para volverse explicita, comprende una dicotomía práctico-teórica y requiere de competencias de auto-reconocimiento.

La práctica educativa busca la resignificación del docente, ya sea de su presente o futuro en función del análisis de hechos pasados, o bien del pasado en función del presente para entender el porqué de alguna situación pasada. Para que haya una resignificación de la práctica el cambio debe ser interno y externo, lo que entendemos como una transformación. Al respecto, Brabàndere (2005), explica que existen dos tipos de cambio: uno perceptual y otro de la realidad. La realidad cambia independientemente de lo que deseamos, en cambio la percepción se modifica conforme a lo que pensamos y creemos; es por ello que para cambiar la práctica del docente se requiere de ambos cambios, de manera que haya una resignificación real y perceptual de la misma.

\subsection{Mediación en la Práctica Reflexiva}

Como seres humanos somos seres sociales y es por esa relación que tenemos con los demás que aprendemos y nos desarrollamos. Estos aprendizajes implican un proceso de mediación e internalización, como es explicado por Vygotsky (Briones, 2006) en su teoría sociocultural. Aunado a lo anterior y a partir de esta teoría, Johnson y Golombek (2011) subrayan que el desarrollo cognitivo es un proceso interactivo mediado por la cultura, la historia, las instituciones, el contexto, el lenguaje y las interacciones sociales. Mientras que, Garza y Leventhal (2000) enfatizan que para aprender requerimos de elementos de mediación tales como el lenguaje u otros signos y símbolos.

Derivado de esta información, se considera que en la práctica reflexiva los docentes deben buscar aprender a partir de lo que viven en su contexto laboral y social, teniendo la oportunidad de reestructurar sus saberes. Es decir, la práctica reflexiva puede desarrollarse si el docente está abierto a recibir y evaluar información proveniente de diversas fuentes como pueden ser de sus colegas, expertos, estudiantes, teorías, otros espacios y su propio medio. Sin embargo, es necesario que, con toda esa información, el docente llegue a actuar como un agente que procede de forma libre e independiente de la estructura en la que se encuentra inmerso.

Un concepto fundamental de Vygotsky para el logro de la mediación es el de Zona de Desarrollo Próximo, el cual es resumido por Johnson y Golombek (2011) como la distancia que existe entre el nivel de desarrollo actual y el nivel al que se quiere llegar y que puede lograrse a través de la mediación o en colaboración con otros. Se requiere de un andamio que es el apoyo que en este caso se le va a dar al docente para promover la reflexión sistematizada en su práctica y que corre a cargo del mediador.

Complementando la teoría sociocultural, se encuentra la teoría de la actividad, que si bien, se origina de una tesis de Karl Marx, es desarrollada por Vygotsky, Leont've y Luria a principios del siglo XX según lo explica Engeström (1999) quien propone un modelo complejo para esta teoría, incluyendo no sólo la actividad (colectiva), la acción (individual) y la operación; sino también elementos como la comunidad, las reglas y la división laboral. Si aplicamos este modelo a la práctica reflexiva podemos encontrar que sus resultados no son únicamente para el docente, sino que habrá un impacto externo que se irá extendiendo. Otra aportación importante de Engeström es el reconocimiento de la externalización, cuando el sujeto es capaz de crear nuevos instrumentos que hacen posible su transformación. Si trasladamos este concepto a la práctica reflexiva, se encuentra que los docentes en un sistema de actividad debieran lograr primero internalizar los conocimientos provenientes del exterior y después externalizar los que ellos concluyan con sus aprendizajes y observaciones. Engëstrom (2010) presenta un modelo de aprendizaje expansivo que puede ser considerado como reflexivo puesto que toma en cuenta el cuestionamiento, análisis, descubrimiento, propuesta de solución, implementación del modelo, reflexión del proceso y consolidación de la nueva práctica; además de ser cíclico.

\subsection{Modelos de Práctica Reflexiva}

Otros modelos reflexivos destacados son el de Argyris y Schön (1974), en el cual proponen comparar y contrastar las teorías en uso (lo que hacemos) con las teorías expuestas (lo que decimos o creemos hacer). Estos autores reconocen dos modelos: 1) El aprendizaje de una vuelta (single loop learning) que consiste en detectar errores $\mathrm{y}$ corregirlos; y 2) El aprendizaje de doble vuelta (double loop learning) que se basa en el cuestionamiento y el cambio de variables para encontrar una solución. Es decir, el primero se enfoca en hacer un cambio operativo, mientras que el segundo busca las causas y consecuencias.

Kolb (Vergara, 2015) presenta un ciclo de aprendizaje experiencial en el que se toman en cuenta las experiencias inmediatas y concretas para ser observadas y reflexionar sobre su significado para crear una o más hipótesis, probarlas y evaluarlas. 
Gibbs (1988) también presenta un modelo cíclico en el que, primero se describe lo sucedido, se identifican los sentimientos que se derivaron de la situación, se evalúa lo que pasó, se analizan los resultados y consultan distintas fuentes para explicarlo, se generan conclusiones y finalmente se toman decisiones de lo que se hará si se presenta un caso similar.

Brookfield (1995) propone tomar en cuenta y analizar cuatro lentes críticos para que el docente reflexione sobre una situación o experiencia: su autobiografía, sus alumnos, sus colegas y las perspectivas teóricas.

Domingo y Gómez (2014) plantean el modelo ATOM que consiste en analizar un incidente y descomponerlo en cada uno de sus elementos divididos en el imaginario del docente, los aspectos curriculares y didácticos, los conocimientos teóricos, las emociones y sentimientos, los aspectos éticos y la intencionalidad, de manera que se identifiquen los aprendizajes obtenidos, su análisis y lo que el docente desea mejorar de su práctica. Además, presentan los modelos R4 y R5 que tienen como finalidad mejorar la práctica, el primero a partir de la reflexión individual y el segundo añadiendo una reflexión grupal. Estos modelos inician con la selección de una situación a analizar, su descripción, la reflexión individual y/o grupal y por último la planificación de la intervención con base en la información obtenida.

Korthagen (2001) formula el modelo reflexivo ALACT de 5 pasos por sus siglas en inglés: action (acción/experiencia), look back on the action (retroproyección), awarness of essential aspects (conciencia de los aspectos esenciales), create alternative methods of action (crear métodos alternativos de acción) y trial (probar su eficacia en una nueva situación).

Esteve, Melief y Alsina (Esteve, 2011) adaptan el modelo de Korthagen para la formación de futuros docentes, dividiéndolo en tres fases: autoanálisis, contraste de ideas con planteamientos teóricoprácticos (fundamentación) y redescripción de los planteamientos iniciales; y seis acciones: verbalización de ideas e inquietudes, contraste de ideas con saberes teóricos, observación del aula y de otras aulas, planteamiento de preguntas de indagación y creación compartida de alternativas de solución.

Pedroza (2014) plantea un modelo de práctica reflexiva de seis etapas: identificación del problema, diagnóstico, fundamentación, supuesto de acción, implementación y teorización o aporte; los cuales deben ser llevados a cabo de forma circular y recursiva, atendiendo a tres procesos: 1) el conocimiento del contexto, de la acción y de la intención; 2) la significación y resignificación de un modelo de acción (puesta en acción, valoración y revaloración); y 3) la emancipación del docente (síntesis de la relación teoría-práctica del nuevo conocimiento al que llega el docente).

Ninguno de los modelos mencionados se contrapone, al contrario, coinciden en una o más de sus partes y en algunos casos se complementan. Es por esa razón que, a partir de ellos, se crea la propuesta para un nuevo modelo de práctica reflexiva en el que se incluyen intencionalmente dos elementos que se considera deben enfatizarse. El primero es el autoconocimiento del docente (o profesionista) en activo y el segundo la participación de un mediador en el proceso, como se explica en el siguiente apartado.

\section{PRÁCTICA REFLEXIVA MEDIADA}

El modelo de Práctica Reflexiva Mediada (MPRM) incorpora tres elementos como su soporte:

1) La mediación de un tutor que acompaña y apoya a los docentes participantes durante todo el ciclo de práctica reflexiva respondiendo a la necesidad de capacitación para el proceso en caso de no haberlo realizado antes o de lo contrario como acompañamiento durante el ciclo promoviendo el andamiaje y acercamiento a la zona de desarrollo próximo; además de la participación de otros agentes como son: otros docentes para promover el intercambio de experiencias y la reflexión colectiva; las aportaciones teóricas de autores expertos en los temas a tratar; y los alumnos por la información que pueden proveer al docente.

2) La búsqueda de autoconocimiento del docente y su práctica como punto de inicio del modelo, en lugar de iniciar a partir de un problema previamente identificado, como lo proponen la mayoría de los modelos. Esto es, se considera que los docentes pueden omitir algunos puntos de su práctica que ya dan por hecho por la experiencia que tienen pero que podrían mejorarse o cambiarse; y

3) La transformación del docente en su actuación real y perceptual, es decir, no se trata de presentar cambios si no está convencido de los mismos; debe haber coherencia entre lo que piensa, dice y hace.

Este modelo se divide en tres fases, cada una con tres ciclos:

Fase 1: Conocimiento del docente y su práctica, a partir de 3 ciclos: 1) un proceso de autoconocimiento por medio de la introspección en el que el docente analiza aspectos de su pasado, presente y futuro principalmente relacionados con los procesos de enseñanza/aprendizaje que ha vivido, está viviendo o 
planea experimentar en un futuro; y en el que se reconozca como ser humano y no sólo como docente., 2) el conocimiento alcanzado a partir de la información que otros le otorguen por medio de las observaciones y consideraciones que hagan de él, de manera que el profesor tenga una idea de cómo lo perciben los demás; y 3) la valoración de la información obtenida en los dos ciclos anteriores al contrastar la información que obtuvo en el primer ciclo (lo que él piensa de sí mismo) con el del segundo ciclo (lo que otros piensan de él) siendo capaz de reflexionar sobre la veracidad de dicha información y teniendo la capacidad de verbalizarla.

Fase 2: Significación de la práctica. Partiendo de los datos obtenidos en la primera fase 1) el docente identifica y plantea un problema o situación significativa y la describe de forma que pueda ser estudiada para su mejora; 2) los docentes buscan información de distintas fuentes confiables y junto con los datos obtenidos a partir de las observaciones y experiencias descritas en la fase uno, los contrastan, de manera que se logre por medio del análisis fundamentar el problema; y 3) se desarrolla un plan de acción que sintetice los aprendizajes de los ciclos anteriores dentro de esta misma fase.

Fase 3: Resignificación del docente y su práctica. 1) el docente lleva el plan diseñado a la práctica, cuidando ser flexible y supervisar como se va desarrollando, al mismo tiempo que mantiene un control de los sucesos por medio de registros y observaciones, 2) se evalúan los resultados de la intervención a partir de todos los datos obtenidos, se reflexiona sobre ellos revalorizando los sucesos y se elabora un aporte teórico; y 3 ) a partir de las acciones y reflexiones, el docente se encuentra en la capacidad de evaluar si hubo alguna transformación no sólo en su actividad sino también mentalmente, es decir si logro resignificar su práctica convencido de ello y no sólo por actuar. En cuyo caso habrá dado un paso hacia su emancipación, entendida esta como su liberación mental convirtiéndose en alguien más crítico y reflexivo.

Una vez terminado el ciclo, se recomienda volver a comenzar, ya sea para tratar otro problema de la práctica o continuar con el mismo en el caso de que los resultados no hubieran sido los esperados y pudieran mejorarse desde otra perspectiva.

El modelo puede sintetizarse a través del siguiente esquema (fig. 1):

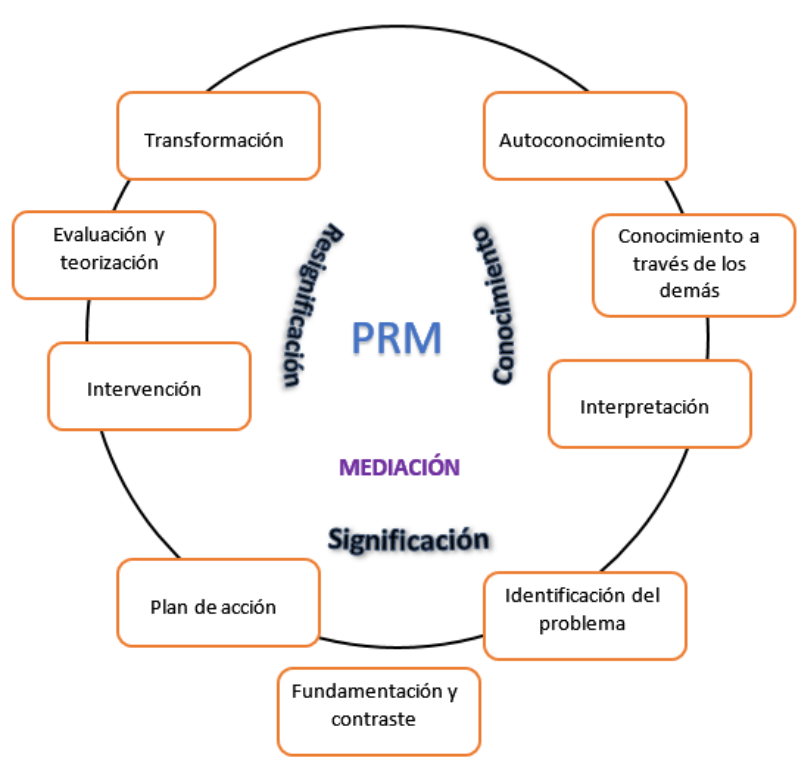

Figura 1. Modelo de Práctica Reflexiva Mediada. Fuete. Adaptado de Cerecero (2016)

\section{MATERIAL Y MÉTODO}

Una vez realizada la investigación teórica y definido el modelo, se diseñó la parte operativa de la investigación, para probar la pertinencia del mismo y poder sustentar su validez. Esta investigación de tipo cualitativa se apoyó en el modelo diseñado y el proceso se comenzó definiendo el contexto de aplicación y los participantes. Por motivos de conocimiento de la institución y facilidades provistas para su realización, el estudio se llevó a cabo con tres docentes del Centro de Enseñanza de Lenguas de la Universidad Autónoma del Estado de México (CELe, UAEM), que decidieron participar voluntariamente a raíz de una invitación directa que se les hizo por cumplir con determinadas características: todos eran profesores en activo, con una trayectoria como docentes superior a cinco años, con estudios de licenciatura o maestría, experiencia de vida en algún país donde se habla el idioma que enseñan, además de contar con documentos que prueban su dominio competente de la lengua. Las sedes principales del CELe de la UAEM se encuentran ubicadas en la ciudad de Toluca de Lerdo, en el Estado de México y cuentan con extensiones en diferentes municipios del Estado, teniendo una matrícula total aproximada de diez mil alumnos por semestre.

Se diseño un taller de capacitación para dar a conocer y poner en práctica el modelo de Práctica Reflexiva Mediada, el cual se llevó a cabo en 6 
sesiones de dos horas cada una, en un periodo de 4 semanas, dejando una semana entre la sesión 5 y 6 para dar tiempo a los docentes de preparar e iniciar su intervención. Una vez concluidas las 4 semanas se dio un seguimiento a los docentes con el fin de observar si presentaban algún tipo de resignificación de su práctica.

El taller se llevó a cabo con una doble intención: recabar datos para sustentar la parte empírica de la investigación y guiar a un grupo de docentes en un ciclo reflexivo mediado para que experimentaran sus posibles beneficios.

El taller presenta una planeación en la que se utilizaron dos sesiones para cada una de las fases y ciclos. Cada sesión incluyó diversas actividades, entre las que encontramos actividades de integración, de introducción al modelo de Práctica Reflexiva Mediada, de autoconocimiento tales como descripciones y charlas de sus experiencias, temas de discusión de los procesos de enseñanza-aprendizaje, análisis FODA, test e incluso la filmación de dos de sus clases para llevar a cabo un proceso de autoconfrontación. También se les pidió llevar un diario de clase en un formato resumido e integrar un portafolio con las actividades del taller, las observaciones de clase y todo aquello que les ayudara a documentar su práctica y hallazgos respecto a la misma; En la tercera y cuarta sesión identificaron un problema relacionado con su práctica, lo describieron y plantearon, buscaron referencias y consultaron a otras personas. Contrastaron y analizaron la información apoyándose del mediador y de sus pares. Prepararon un plan de intervención, lo pusieron en práctica y presentaron un reporte con los resultados parciales en la última sesión, teniendo una participación individual frente al grupo y una reflexión grupal; a partir de la última sesión, los docentes siguieron teniendo el apoyo del mediador y revisiones periódicas durante el siguiente mes.

La viabilidad de la investigación fue posible en todos sus aspectos, generando costos mínimos de papelería y un porcentaje de trabajo extra para los docentes participantes de manera que pudieran ejecutar todas las actividades y la filmación de su clase al inicio y al final del ciclo.

El portafolio de cada docente, las filmaciones de clase y de cada una de las sesiones del taller, las notas y observaciones fueron utilizadas por el investigador para hacer el metaanálisis de los procesos y resultados presentados por los participantes.

\section{RESULTADOS}

Situándose en el modelo de Práctica Reflexiva Mediada, el análisis de los resultados fue obtenido por medio de nueve categorías, cada una correspondiendo a uno de los ciclos del modelo: introspección, consideración, verbalización, descripción, análisis, síntesis, acción, revaloración y emancipación. Entre los hallazgos encontrados están los siguientes:

Los participantes aplican, consciente o inconscientemente, conceptos fundamentales para su práctica que provienen de su formación y experiencia y que no necesariamente coinciden con sus teorías en uso. Es decir, retoman sus experiencias pasadas para explicar lo que sucede en el aula y se les dificulta introducir las nuevas tendencias en su práctica o realizar cambios que vayan de acuerdo con su situación actual, aun cuando manifiesten lo contrario.

A partir de los documentos, observaciones y videos se pudo determinar el estilo de aprendizaje y docencia de los profesores. Sin embargo, por el número de participantes, no fue posible corroborar si uno de los estilos de aprendizaje pudiera ser considerado dominante para los profesores de lenguas, en el caso presentado.

Durante el proceso de autoconfrontación los docentes se enfocaron principalmente en sus alumnos, lo que se atribuye a la dificultad de hablar de sí mismos y auto-observarse, como si ello los liberara de la responsabilidad de darse cuenta como son y como se ven ante los demás, por una parte; y como pueden actuar como agentes e influir en los procesos de aprendizaje de sus alumnos, por otra.

Los profesores sujetos de estudio, en repetidas ocasiones, mostraron dificultad en distinguir entre sus percepciones y sus observaciones, lo cual interpretamos como una falta de reflexión, per se, o bien una carga emocional fuerte.

Los docentes, al saberse observados durante la filmación de clase, actuaron de la manera en que ellos pensaron era lo correcto; sin embargo, mostraron cierto nerviosismo delatado por su lenguaje corporal. Al observarse en el video, con el acompañamiento del mediador, fueron capaces de expresar como se visualizaban y explicar el porqué de sus acciones, sus creencias y dar datos con mayor claridad y confianza.

Por medio de los formatos y actividades del taller se logró obtener información de cada docente en cuanto a sus hábitos, creencias, sentimientos, expectativas, valores, experiencias, conocimientos, actitudes, entre otros.

Todos los participantes eligieron definir un problema relacionado con los procesos de aprendizaje, orientándose en cómo podrían ayudar a sus alumnos. 
La búsqueda de información sobre el tema que cada docente eligió fue principalmente bibliográfica o documentada de internet, partiendo de sus conocimientos previos y costándoles un poco más de trabajo encontrar documentos serios con ideas nuevas o contrarias a lo que ellos esperaban.

La planeación de la intervención fue parcial. Los logros fueron relativos dependiendo el grado de involucramiento del docente y su interés. Uno de los planes de acción se tuvo que reestructurar al descubrir nueva información respecto a determinados alumnos, aplicando la nueva información obtenida.

En cuanto a la aplicación del modelo, se consiguió llevar a cabo un breve ciclo de práctica reflexiva completo. No obstante, los participantes manifestaron su agrado hacia el modelo y aceptaron su utilidad manifestando la necesidad de una continuidad y de otorgar mayor tiempo para el proceso.

Se logró la unidad y participación del grupo y se observó que los participantes aportan más información de forma verbal durante el taller que en los documentos escritos.

Los docentes presentaron una aportación teórica corta lo que ha sido atribuido 1) a lo breve del ciclo reflexivo al que fueron expuestos y 2) a un proceso no consolidado en el que se logró un grado parcial de internalización del conocimiento y un menor grado de externalización.

Si bien, la práctica reflexiva puede ser llevada a cabo por un solo individuo, el acompañamiento de un mediador y de los pares puede facilitar el proceso, acrecentar la motivación, crear una red de apoyo y ampliar las posibilidades de obtener ideas e información que cuando se actúa en soledad, además de asegurar hasta cierto punto la continuidad del proceso y el logro de resultados.

\section{DISCUSIÓN}

Como lo menciona Melief, et. al. (2010), el aprendizaje es un proceso social e interactivo. A partir de esta investigación fue claramente identificada la importancia de la mediación a través de un mediador, distintas fuentes y la participación de otros agentes como son los pares y alumnos; todo esto como parte de un proceso social para el aprendizaje del docente respecto a si mismo y su práctica.

Por otro lado, John Dewey (1993) y Latorre (2003) afirman que uno de los pasos en la reflexión implica investigar para generar un diálogo entre la teoría y la práctica. Esto es cierto, en tanto sea una reflexión y no una pre-reflexión. Por tanto, la investigación debe formar parte integrante de la práctica reflexiva.
Otro elemento importante que considerar en la práctica reflexiva es la verbalización al inicio del ciclo reflexivo y durante la teorización, puesto que constituye un instrumento básico para comprender el grado de internalización y externalización alcanzadas. Como lo menciona Onrubia (1993) el lenguaje es un instrumento esencial en la ZDP porque permite reorganizar y reestructurar los conocimientos.

Zeichner (1982) argumenta que la reflexión en la práctica no debe ser aplicada únicamente a aspectos técnicos porque puede provocar una ilusión de lo que es, utilizándola como pretexto político para que se reproduzcan determinadas prácticas en lugar de que los profesores desarrollen sus propias teorías. En la investigación se observó que un problema común entre los participantes fue que cuando se les preguntaba su opinión sobre la mejor pedagogía y procesos de enseñanza-aprendizaje, ellos contestaron en general la información que corresponde a las tendencias actuales al respecto, sin que necesariamente lo apliquen o lo crean; es decir, en un inicio mostraron una reflexión técnica en la que muestran sus conocimientos teóricos aceptando la teoría como ha sido construida, de forma acrítica e irreflexiva, dejándose, en ocasiones, llevar por las tendencias de las investigaciones y sin darse tiempo para cuestionarlas. Acerca de este problema, tanto Carr (1996) como Zeichner (1982) advierten que los profesores se han convertido en consumidores o clientes de las investigaciones que se publican.

Aunado a lo anterior se observa que no siempre hay concordancia entre lo que se piensa, se dice y se hace, por lo que la propuesta de Argyris y Schön (1974) para identificar las teorías en uso y las expuestas, así como contrastarlas, puede disminuir este problema.

La Práctica Reflexiva Mediada no va en contra de ninguno de los modelos mencionados pero si retoma como puntos de partida un aprendizaje realista, como es entendido por Melief, Tigcheelar y Korthagen (2010); el conocimiento (aunque no sólo de un problema), la significación y resignificación de la práctica como los contempla Pedroza (2014); y una co-construcción de conocimientos dentro de un proceso dialógico y dialéctico entre teoría y práctica por medio de la mediación como es entendido por Esteve (2011).

\section{CONCLUSIONES}

Las aportaciones principales de esta investigación a través del MPRM están encaminadas a la 
formalización del uso de la mediación como una herramienta que facilite la reflexión en la práctica.

Aunado a lo anterior se hace énfasis en otorgar un tiempo para el autoconocimiento del docente y de su práctica, con la finalidad de poder darse cuenta de situaciones que de lo contrario y debido a la cotidianeidad pudieran pasar desapercibidas además de aceptar que los procesos de enseñanza aprendizaje son complejos y están en constante cambio debido a distintos factores como son la época y el contexto.

Existe cierta disonancia entre lo que los profesores hacen y lo que dicen. En general, hablan de una práctica actual de acuerdo con lo que consideran se espera de ellos, pero en realidad actúan de acuerdo con su edad, tiempo, experiencias y circunstancias de vida. Sus experiencias como estudiantes y como docentes influyen fuertemente en la forma en que construyen sus creencias y conceptos.

Normalmente, se enseña a partir de un contexto determinado, en el cual consciente $o$ inconscientemente se hacen modificaciones a partir de las distintas percepciones, experiencias, características de los actores, necesidades de los grupos y de las instituciones. Es por ello, que es necesario reflexionar sistemáticamente para no dejar a la suerte los resultados obtenidos.

Otro punto importante que retoma el MPRM es la importancia de la investigación a través del reconocimiento de una relación dialógica y dialéctica entre la teoría y la práctica.

Un problema que se ha presentado en la práctica reflexiva es el enfoque técnico que en algunos casos se le ha dado y que a través de la PRM se pretende subsanar al acentuar ese proceso de interacción con otros y consulta de distintas fuentes incluyendo aquellas contrarias a las ideas preconcebidas $o$ conocimientos previos del docente para dar oportunidad a su emancipación mental.

El MPRM también contrae el compromiso de asumir su responsabilidad para atreverse a ser agentes activos, dispuestos a cambiar situaciones del aula en lugar de responsabilizar a otros de los problemas que se le presenten.

Las observaciones únicamente son posibles si se quita intencionalmente la carga emocional que conllevan las percepciones.

El lenguaje corporal juega un papel importante para ayudarnos a entender la coherencia entre lo que se piensa, dice y hace.

De la actitud del docente y su grado de compromiso depende la profundidad y los logros alcanzados a través de la PRM.

Cuando los docentes comparten sus experiencias se crea un ambiente de confianza y compañerismo; los profesores se dan cuenta de que no están solos, que muchas de las cosas que pasan en su aula son comunes en las otras y que pueden ayudarse al intercambiar ideas y prácticas que han probado.

Finalmente, se define a la Práctica Reflexiva Mediada como un proceso de reflexión sobre la praxis del docente y los factores que influyan en ella; el cual debe ser continuo, intencional y sistemático y llevarse a cabo en compañía de un mediador y otros agentes de apoyo, comenzando por un proceso de autoconocimiento y conocimiento del medio, en el cual se toma en cuenta la experiencia, la práctica, la teoría y el contexto para poder hacer un juicio razonado y reflexivo que ayude a la toma fundamentada de decisiones y al desarrollo personal y profesional del docente que la ejerce.

\section{REFERENCIAS}

Aguilar Mejía, E., y Viniegra Velázquez, L. (2003). Atando teoría y práctica en la labor docente. México: Paidós Educador.

Argyris, C., y Schön, D. (1974). Theory in Practice. San Francisco: Jossey-Bass.

Atkinson, S. (Ed.). (2011). El libro de la Filosofía. Madrid, España: Ediciones Akal/Darling Linderley Limited.

Barnett, R. (1992). Improving Higher Education. Buckinham: SRHE/Open University Press.

Berté, M. (2014). Reflexionar: Un modo mejor de pensar. Madrid: Narcea.

Brabàndere, L. (2005). The forgotten half of change: Achieving greater creativity through changes in perception. New York: Diversion books.

Briones, G. (2006). Teorías de las ciencias sociales y de la educación: Epistemología (2a. ed.). México: Trillas.

Brookfield, S. D. (1995). Becoming a critically reflective teacher. San Francisco, CA: Jossey-Bass.

Carr, W. (1996). Una teoría para la educación. Madrid: Morata.

Cerecero Medina, I. E. (2016). Teorización de los procesos de resignificación de la práctica educativa del docente de lenguas. Universidad Autónoma del Estado de México, Toluca. Recuperado a partir de https://ri.uaemex.mx/bitstream/handle/20.500.11799/6 5347/TESIS\%20Ingrid\%20FINAL-splitmerge.pdf? sequence $=3$

Delval, J. (1999). Los fines de la educación (7a. ed.). México: Siglo veintiuno editores.

Dewey, J. (1993). Como pensamos: Nueva exposición de la relación entre pensamiento y proceso educativo. (A. C. Barcelona), Trad.) Barcelona: Paidós.

Dewey, J. (1995). Democracia y educacion: Una introducción a la filosofía de la educación (2a ed.). Madrid: Ediciones Morata.

Domingo Roget, Á., y Gómez Serés, M. V. (2014). La Práctica Reflexiva: Bases, modelos e instrumentos. Madrid: Narcea.

Elliot, J. (2000). El cambio educativo desde la investigaciónacción (3a. ed.). Madrid: Ediciones Morata.

Engëstrom, Y. (2010). Activity theory and learning at work. (U. o. Helsinky, Ed.) Obtenido de Center for Activity Theory and Developmental Work Research: 
http://www.helsinki.fi/cradle/documents/Engestrom\% 20Publ/Chapter\%20for\%20Malloch\%20book.pdf

Engeström, Y., Miettinen, R., y Punamaki, R.-L. (1999). Perspectives on activity theory. NY: Cambridge University Press.

Esteve, O. (2011). 2. Desarrollando la mirada investigadora en el aula. La práctica reflexiva: Herramienta para el desarrollo profesional como docente. En U. R. Coordinador Bikandi (Ed.), Lengua castellana y literatura. Investigación, innovación y buenas prácticas. (Vol. III, págs. 29-48). Barcelona: Grão.

Gadamer, H.-G. (1980). Practical philosophy as a model of the human science. Research in Phenomenology(9), 74-85.

Gallagher, S., y Zahavi, D. (2013). La mente fenomenológica (2a. ed.). España: Alanza Editorial.

García, A. D. (1999). Introducción a la fenomenología de Edmund Husserl. Obtenido de Revista de ciencias humanas:

http://www.utp.edu.co/ chumanas/revistas/revistas/re v22/garcia.htm

Garza, R. M., y Leventhal, S. (2000). Aprender como aprender (3a. ed.). México: Trillas/ITESM.

Gibbs, G. (1988). Learning by doing: A guide to teaching and learning methods . Oxford: Oxford Brookes University.

Johnson, K. E., y Golombek, P. R. (2011). Research on second language teacher education: A sociocultural perspective on professional development. New York: Routledge.

Kemmis, S. (1988). El curriculum: más allá de la teoría de la reproducción (3a. ed.). Madrid: Ediciones Morata.

Korthagen, F. (2001). Linking practice and theory. The pedagogy of realistic teacher education. Londres: Lawrence Erlbaum Associates.

Latorre, A. (2003). La investigación-acción: Conocer y cambiar la práctica educativa. Barcelona: Gráo.

Melief, K., Tigchelaar, A., y Korthagen, F. (2010). Aprender de la práctica. En Creando mi profesión: Una propuesta para el desarrollo profesional del profesorado (págs. 19-38). Barcelona: Octaedro.

Pedroza, R. (2014). La Investigación-Acción en la Práctica Educativa Reflexiva ( 2 a ed.). México: Colegio de Investigadores en Ciencias de la Educación.

Schön, D. A. (1992). La formación de profesionales reflexivos: Hacia un nuevo diseño de la enseñanza y el aprendizaje en las profesiones. Barcelona: Ediciones Paidós y Ministerio de Educación y Ciencia.

Schön, D. A. (1998). El profesional reflexivo: Cómo piensan los profesionales cuando actuan. Barcelona: Paidos.

Schwab, J. J. (1983). "Un lenguaje práctico como lenguaje para el curriculum". En J. Gimeno Sacristan, y A. Pérez Gómez, La enseñanza: su teoría y su práctica (págs. 197-209). Madrid: Akal.

Tardif, M. (2004). Los saberes del docente y su desarrollo profesional. Madrid: Narcea.

Vergara, C. (2015). Actualidad en psicología. Obtenido de http://www.actualidadenpsicologia.com/la-teoria-delos-estilos-de-aprendizaje-de-kolb/

Zeichner, K. M. (8 de mayo de 1982). "Factors related to reading performance". Obtenido de Práctica Reflexiva: http://www.practicareflexiva.pro/wpcontent/uploads/Org-El-maestro-como-profesionalreflexivo-de-Kenneth-M.-Zeichner..pdf 